\title{
Protée
}

\section{Ceci n'est pas le corps de Chouinard}

\section{Isabelle Ginot}

Volume 29, numéro 2, 2001

\section{Danse et altérité}

URI : https://id.erudit.org/iderudit/030628ar

DOI : https://doi.org/10.7202/030628ar

Aller au sommaire du numéro

\section{Éditeur(s)}

Département des arts et lettres - Université du Québec à Chicoutimi

\section{ISSN}

0300-3523 (imprimé)

1708-2307 (numérique)

Découvrir la revue

\section{Citer cet article}

Ginot, I. (2001). Ceci n’est pas le corps de Chouinard. Protée, 29(2), 77-84.

https://doi.org/10.7202/030628ar

\section{Résumé de l'article}

Le corps dansant du Faune de Marie Chouinard est un corps multiple, où se superposent des images, des histoires et des matérialités corporelles différentes et contradictoires. Habité de formes et de poncifs concernant tant le Faune de Nijinski que les canons de la danseuse, du masculin, du féminin, de l'humain, de l'animal, du naturel et de l'artificiel, il fait surgir également les poncifs qui organisent le regard et le discours de celui qui l'observe. Peut-on penser ce que l'on regarde quand les frontières entre organique et prothèse corporelle ont été dissoutes?
Ce document est protégé par la loi sur le droit d'auteur. L'utilisation des services d'Érudit (y compris la reproduction) est assujettie à sa politique d'utilisation que vous pouvez consulter en ligne.

https://apropos.erudit.org/fr/usagers/politique-dutilisation/ 


\section{CECI N 'EST PAS LE CO RPS DE CHOU IN ARD 1}

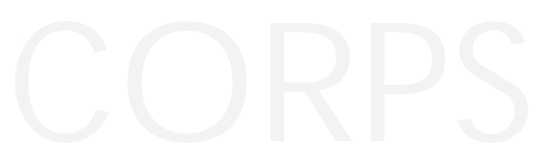

ISABELLE GINOT

Voilà un objet qui doit bouillir à haute température pour être bien propre, qui peut être donné en cadeau, jeté à la poubelle ou servir de presse-papiers. L'amour s'en va, l'amour revient, les partenaires sexuels vont et viennent mais le gode est toujours là. Il est le survivant de l'amour. $^{2}$

Mufle offensif, deux cornes de bouc: l'une, tournée vers l'arrière, s'enroule sur elle-même; l'autre se tend toute droite vers l'avant. La bouche s'agite, vorace ou torturée, la mâchoire projetée tire sur la nuque, lèvres actives laissant échapper un discours silencieux ou happant quelque objet désirable. Le ventre plaqué, avalé sous la cage, est le nœud remuant d'un conflit entre le bassin bloqué en position frontale, et la cage thoracique vissée face au public, suspendue aux bras perçant l'espace, dessus, devant, tendus vers quels objets? Mais le ventre plein de remous tire vers le bas tout ce qui veut s'étendre vers le haut, résistant à l'aérien de toute sa force terrienne. La cuisse droite est renflée et s'ajuste mal au bassin, la hanche noyée dans trop de chair; la cuisse gauche, plus fine, laisse échapper des fibres sombres, poils clairsemés, épines menaçantes ou flèches plantées dans la chair. Les pieds et les jambes regardent vers l'avant (la coulisse jardin) ou font demi-tour pour fixer l'autre bord de scène tandis que le buste, cloué face au public, ignore pour un temps le changement d'orientation.

\section{SAINTE CHOUINARD}

Cette description en forme de blason pourrait être celle d'un amoureux (pourrait-elle être celle d'une amoureuse?) ou le trajet en gros plan d'une caméra dévoilant les détails du corps de «la danseuse». Elle signalerait l'engagement du regardeur (peut-être pas encore voyeur) dans un érotisme du fragment (que l'on dispenserait, pour une fois, du soupçon de fétichisme). Elle s'appliquerait, dans un mouvement d'approfondissement, à presser le signe pour en extirper le sens, se saisirait de l'agitation des entrailles, de la tension énergétique, de la violence-rentrée du geste pour accoucher des émotions, dévoiler le lien indissoluble entre geste-et- 
sens, vérité-du-mouvement et profondeur-esthétique; elle pourrait également partir de là pour consolider cette hypothèse, malheureusement encore mal acquise, selon laquelle il n'y a pas de corps, mais des sujets, du sens qui fait geste et du geste qui fait sens. Que la danse est cet art qui impose de repenser la coupure judéo-chrétienne entre corps et âme, pour imposer la notion d'un sujet intègre et cohérent. Enfin, prenant en compte tant la psychanalyse que nombre de pratiques chorégraphiques, elle considérerait la déchirure de ce sujet et les forces contradictoires qui s'affrontent dans son geste, signant l'emprise de forces incoercibles sur le désir du sujet dansant que l'on identifierait, c'est selon, des noms de l'interprète, de la chorégraphe, ou encore du Faune.

Dans cette optique, une hagiographie (de la danseuse? de Chouinard? du Faune?) s'impose: en rendant hommage à l'œuvre de Nijinski (dont l'empreinte est plus qu'évidente dans la pièce composée à partir des poses de Nijinski, capturées dans un célèbre album de photos du baron de Meyer), Chouinard paie le nécessaire tribut à une ouvre considérée comme fondatrice de toute la modernité en danse. Ce faisant, et tout comme Nijinski en son temps (preuve supplémentaire de la valeur de son hommage), elle rend le corps, à travers la figure du Faune, à sa force pulsionnelle non entravée, au flux avoué et multiple de son désir. Elle réhabilite un corps dansant délivré des coupures de la perspective, du modèle aérien toujours prégnant de la danse classique; elle rend au mouvement les parties honteuses du sexe, des entrailles, du cour, abandonne les canons morphologiques de la danseuse au profit d'une véritécorporelle-enfin-dévoilée. Davantage encore, elle affiche la souffrance du désir, l'ambivalence d'un sujet pris non seulement entre humanité et animalité, mais aussi entre féminin (les seins, le ventre, la gorge offerte, la bouche ouverte) et masculin (les cornes, le pénis, la cuisse puissante, la pénétration). «Le corps» du FauneChouinard a les entrailles en pleines marées, son-gesteplonge-dans-les-profondeurs-de-sa-mémoire, il-fait-remonterdans-le-monde-du-visible-les-couches-enfouies-de-notre-mémoirearchaïque, il-ondule-au-flux-d'une-nature-enfin-libérée, qui- partage-avec-le-monde-animal-les-liens-renoués-avec-les-originesdu-vivant. Pour preuve, le bruit du souffle, qui circule bruyamment du dedans au dehors - discrètement médiatisé par quelque merveille des technologies HF et rend tangible cette vie-intérieure du corps en tant qu'elle échappe à toute représentation: le viscère comme origine indépassable du geste. "Dans ses solos, le mouvement semble frayer sa voie depuis le "silence" du corps viscéral» ${ }^{3}$. "Je voulais sentir que je respirais un air chauffé à blanc, saturé, que chaque respir me dilatait, augmentait les croissances internes » ${ }^{4}$.

\section{MAUdiTE CHOUINARD}

Dans la même optique, pourtant, il faudra aussi dénoncer Marie Chouinard. L'Après-Midi d'un Faune, créé en 1987, fut présenté en 1999 dans un programme réunissant tous les solos de Chouinard (1978-1998) interprétés par quatre danseuses différentes et interchangeables puisque plusieurs de ces solos apparaissent, sur le programme, interprétés par "Carole Prieur ou Marie-Josée Paradis» (pour Le Faune, autres choix possibles pour d'autres solos). L'échangisme n'est pas tout à fait un péché mortel en danse, mais il fait naître un soupçon, particulièrement en France au moment de cette programmation: il règne alors dans la profession une idéologie sanctifiant l'interprète, son statut créateur, sa singularité, la violence des effets sur lui des rapports de pouvoir avec le chorégraphe, le public, les institutions, etc. Cette idéologie s'est constituée en résistance à une autre, qui entretenait l'invisibilité du danseur-interprète au profit de la toutepuissance du chorégraphe-auteur. Le caractère remplaçable de l'interprète ne peut donc se justifier que par l'intention de faire émerger plus encore les singularités, la nature exceptionnelle et authentiquement créatrice de chaque danseuse ${ }^{5}$. Or, les différentes interprètes qui se succèdent au fil des solos semblent toutes des clones (de Marie Chouinard, supposera-t-on immanquablement, même si, comme c'est mon cas, on n'a jamais rencontré la chorégraphe). L'effet de clonage est décuplé par un amoncellement de postiches et prothèses. Marie Chouinard serait donc une chorégraphe diabolique: elle rejetterait l'altérité de 
l'interprète, ferait de ses danseuses de fidèles miroirs; on sait ce que ces jeux de miroir de maître à élève, ou d'interprète à chorégraphe (n'est-ce pas presque toujours la même chose?) doivent aux jeux d'emprises non choisies, entretenus par les rapports de pouvoir si bien décrits par Foucault ${ }^{6}$. Double faute, d'ailleurs: non seulement elle entretient avec ces interprètes ce coupable rapport de clonage, mais encore, elle manque son but: les connaisseurs (ceux qui ont vu Chouinard danser elle-même les solos) sont déçus, et témoignent du manque qui nous sépare de toutes les origines. L'interprétation n'est qu'un mime laborieux de ses gestes, privés du lien (du souffle) qui les rattachait originairement au sens: à l'imaginaire de Chouinard, son intériorité, bref, sa présence?

\section{CECI N'EST PAS UNE PIPE}

Le statut borderline de la prothèse est significatif de

l'impossibilité de tracer des limites nettes entre le «naturel» et

"l'artificiel», entre le "corps» et la «machine». La prothèse

montre que la relation corps/machine ne peut pas être

simplement comprise comme un assemblage de parties discrètes

articulées ensemble de manière à accomplir une tâche spécifique. ${ }^{8}$

Séquence connue dans Le Dernier Spectacle de Jérôme Bel (1998): chaque danseur à son tour entre en scène, annonce «je suis Susanne Linke», et danse un fragment de Wandlung, de Linke, sur une musique de Schubert. La question posée par Chouinard est la même que celle de Bel, mais selon une méthode inverse: elle force l'identification (les danseuses sont des clones) tout en rendant impossible la (con)fusion: en exposant l'interchangeabilité des danseuses, elle trouble et démultiplie les origines-de-la-danse. À propos de la pièce de Bel, André Lepecki pointe la dissolution «des rapports de propriété entre corps, sujet, image corporelle et nom» et interroge «un discours qui invente le fantasme d'un sujet-monade contenu à l'intérieur d'un corps toujours perçu comme une image " ${ }^{9}$. Est-il possible de voir/penser la danse en dehors de la croyance qui nous fait confondre "le/la chorégraphe» (ou "l'interprète»), sa subjectivité, et son corps? Peut-on voir «un corps» comme un objet, autonome et indépendant du geste, de l'intention, de la subjectivité... Quelle danse émergerait alors? Peut-on décoller la danse de la danseuse, l'œuvre du chorégraphe, le geste du corps?

Il est peut-être temps d'essayer de penser le corps et l'interprète comme des objets qui peuvent "être donnés en cadeau, jetés à la poubelle ou servir de presse-papiers». Objets eux aussi dégagés de tout rapport de hiérarchie: objets non plus d'un sujet, mais indépendants de tout sujet. En pulvérisant les catégories de chorégraphe, d'interprète, d'œuvre et de corps, on pourra peut-être du même coup faire sauter les rapports d'emprise qui verrouillent leurs relations. Si les catégories sont mortes, peut-être corps, objets, danses, sujets pourront-ils se remettre à danser.

Le corps de Chouinard est à la fois absent et obscènement omniprésent: il n'y a pas de vrai-corpsChouinard. Le corps du Faune est à la fois de chair, et d'artefacts: il n'y a pas de vraie-chair. Peut-il y avoir encore un corps d'interprète s'il n'y a pas de corps de chorégraphe ${ }^{10}$ ? Le Faune de Chouinard pourrait bien être une prothèse d'un autre espace, non linéaire (malgré l'espace chorégraphique en couloir de la pièce) où «la danseuse» n'est en rien l'instrument, l'expression, le sujet d'une chorégraphe, mais la prothèse-objet d'une danse.

\section{TRANSGENRES}

Comme son Faune frémissant qui ne parvient à fixer son élan dans aucune direction, Chouinard éveille et agace mon esprit. Ma pensée organise une lecture en fonction - valeurs discutables, mais assumées - des critères-d'évaluation dont une partie au moins sont explicites. Exemples: la suggestion ou l'évocation sont des valeurs positives, au contraire de la pléthore ou du trop-plein qui ont un effet autoritaire sur la perception du spectateur. L'excès de puissance corporelle est l'objet de tous mes soupçons: il signe le privilège du quantitatif sur le sensible, et s'accompagne presque toujours de valeurs associées au machisme (qui abondent sur les scènes françaises). Le corps sensible, visible et peu apprêté attire ma sympathie plus que les harnachements et costumes 
imposants, car il fait place à l'expérience de la danse plus qu'à la production d'images. Enfin, la place de l'interprète comme sujet autonome est un aspect essentiel de mon plaisir comme de mon jugement esthétique. Mais un désir s'éveille qui bute sur ces catégories. Quelque chose en moi aime dans Chouinard ce que mon esprit rejette, et par là même décale ma pensée et la rend inopérante. J'aime ces (trop rares) états de conflits de la sensibilité où le désir vient troubler l'autorité (sur moi-même et sur les autres) de mon discours critique. Mais est-il permis de parler du désir de la critique?

Trois heures de corps musculeux en grand apparat, coiffures, maquillages identiques. Je me perds dans les interprètes, revenant constamment au programme qui ne m'apprend rien si ce n'est que je vois actuellement "ou... ou...», que je suis incapable de distinguer de «..., ou ...». Postiches, prothèses, excroissances,

monstruosités s'accumulent, corps déformés, reformés, augmentés dans une outrance que nous épargnent, habituellement, les scènes françaises dites d'avantgarde. Chouinard opère sur le mode de l'invasion, de la pléthore et du trop-plein; elle défigure les corps par l'adjonction de prothèses en tout genre (mais toujours d'aspect obscènement phallique). Images et associations, désirs et rejets s'amoncellent en une "compression" de danses totalement dépourvues d'espaces vides. Chouinard restera d'abord, pour moi, un cyborg de supermarché, définitivement associée à un feuilleton télévisé: les corps mutants de Stargate, ses rites initatiques médiévaux, ses antres préhistoriques abritant les technologies de pointe les plus maléfiques, ses fantasmes de surpuissance. Que penser de cette association d'une pièce chorégaphique contemporaine avec ce standard de la culture populaire américaine de mauvais goût?

Une longue tradition de la danse moderne nourrit le fantasme de la reconquête d'un corps-naturel, dont les formes multiples empruntent inévitablement les motifs du retour vers un corps-originel (de l'enfant, d'avant la civilisation, de l'animal, etc.), de la redescente vers quelques profondeurs, ou encore de la mise-à-nu (au propre comme au figuré) d'une vérité-du- corps perçue comme au-delà, ou en dessous, des surfaces cultivées (gestes conventionnels, vêtements, etc.). Il-était-une-fois-la-vérité-du-corps. Mais cette vérité s'accommode mal des appendices ostensiblement artificiels dont ce corps-ci abonde. La palpitation des naseaux du Faune, l'agitation de ses intérieurs, l'excitation de sa surface cutanée sont autant de signaux d'une danse qui échappe au discours (unedanse-d'avant-le-langage), et nous plonge dans les espaces bienheureux d'avant-les-coupures-de-la-langue, où-le-sens-n'apas-encore-vécu-les-mutilations-du-signe. La-danse-des-origines opère par définition de façon suggestive, elle procède par ondes et non par explicitations. Que faire des cornes qui ridiculisent, en l'extériorisant, le travail intérieur de l'animalité? Que faire des simulacres d'un corps postiche imité de Nijinski, qui se moquent de l'essence du corps et du geste? La recomposition d'une morphologie ostensiblement (et exagérément) imitative ne renvoie-t-elle pas à la primauté du visible sur le sensible, au pouvoir d'un corps idéal (même si ce corps-ci s'oppose aux canons du corps dansant classique)?

Chouinard affiche une organicité bruyante, mais assistée par orthopédie. Le souffle continu et sonorisé informe le public de l'effet sensuel en feed-back que produit la danse "de l'émoi» ${ }^{11}$ sur la danseuse, mais ce raffinement sensitif porte tous les signes grand public d'une esthétique sado-masochiste (métal, chaînes, cuirs, objets prosthétiques et godes en tous genres). Elle conte un état subtil où désir et jouissance dialoguent dans une prise délicate avec l'espace, mais exhibe un corps surpuissant, plus conforme aux canons du body-building qu'à ceux de la danse contemporaine (surtout française, qui privilégie les modes du sensible, de la défaillance, voire de l'effondrement). Le corps de Chouinard active des idéologies contradictoires de la danse; il exhibe en même temps tous les signes du sentir-authentique, et des prothèses qui ne tentent même pas (comme toute prothèse devrait s'y efforcer) de se faire discrètes, d'imiter au mieux la nature et ses fonctions. La prothèse le déforme et le morcelle à la fois en «essences» différentes (un bout de Nijinski, un bout 
de Faune, un bout de femme, un bout d'homme...), et en "genres» (poncifs) hétérogènes: danse organique, science-fiction, récit mythique, performance d'avantgarde, sitcoms... bon goût et mauvais goût. Il est multiplement monstrueux et multiplement transgenre. Sa monstruosité n'est pas seulement celle d'un corps homme-femme, humain-animal, organique-artificiel; elle heurte aussi l'homogénéité de catégories de genre aussi inébranlables que les évidences de la "différencedessexes» 12 : les genres esthétiques.

\section{OBSCÉNITÉS}

Le Faune détache d'un geste sec une corne, la brandit comme un poignard (un pénis) vers le ciel, puis se l'attache (velcro? pression? crochet? aimant?) au pubis. En guise des nymphes que le Faune de Nijinski voit en rêve et dont le souvenir excite le désir, des traits verticaux de lumières s'allument devant le Faune-Chouinard, qui les éteint par le geste même qui veut les pénétrer - une avancée crispée du bassin armé de son gode-corne. L'intensité de l'expérience dansée défaille devant le ridicule du signe. Je reconnais ou j'invente, au moment de ces coïts impossibles d'une corne avec un rayon de lumière, le son pathétique d'un moteur de camion échouant à démarrer. La bande-son ancre pour moi le sentiment d'un double ou triple discours, une ironie difficile à identifier tant le geste lui-même semble se prendre au sérieux; elle met à distance le drame de l'absence ou de l'évanouissement de l'objet du désir, de la tension extrême du corps porte-gode en mal de décharge ${ }^{13}$. Le Faune décroche sa corne pour se la raccrocher ailleurs: le corps-organique s'affiche comme corps détachable, aux organes remplaçables et démontables, aux fonctions interchangeables (le déplacement de la corne ne modifie pas radicalement la nature du geste du Faune). La-vérité-du-geste, l'intégrité-du-corps, lecaractère-originaire-de-l'œuvre-de-référence (le Faune de Nijinski): un kit de danse. Plus que la corne-gode, ce qui se démembre là c'est l'origine de la danse (le fait même qu'il y ait une origine), l'homogénéité de «la danse moderne» et des catégories qui me permettent de la penser. Il faudrait donc retirer au Faune-
Chouinard ses excroissances amovibles pour restaurer la-vérité-de-la-danse-et-du-corps ${ }^{14}$.

Tous les codes (les godes?) esthétiques qui dessinent le corps du Faune le travaillent en force. Cette danse ignore l'euphémisme: aucune métaphore, mais «la chose» elle-même, les choses surtout, qui se surimpriment et se contredisent. La crudité des images qu'il produit (non suggestives, non elliptiques, non ambiguës) impose une sévère fermeture du signe: le Faune-Chouinard enferme le regard, mais dans plusieurs espaces simultanés et séparés. Voir sa danse aura été, pour moi, cette schizophrénie du regard qui décompose en retour l'homogénéité de ma propre perception. Elle pulvérise les catégories esthétiques qui organisent mon regard et dérègle les adhérences entre le corps (le sien, le mien) anatomique et le corps désirant, elle refait d'autres anatomies du corps, de la danse et du spectacle.

\section{ÉTATS DE CORPS, ÉTATS DE gode}

Le cyborg n'est pas un ordinateur mais un être vivant branché à des réseaux visuels et hypertextuels qui passent par l'ordinateur de telle manière que l'être vivant connecté devient sa prothèse pensante. 15

Le corps de Nijinski déborde du corps de Chouinard-Prieur, épaissit une cuisse, exagère un mollet. Les muscles de la danseuse se bandent-ils pour résister à leur propre désintégration sous la poussée du maître, ou pour se mettre au niveau de sa légendaire intensité? Le Faune-machine expose crûment son caractère composite par des couches hétérogènes qui se découvrent mutuellement sans que jamais ne soit révélé un corps-du-dessous plus authentique que les autres. Cela commence avec les postiches qui reforment les contours fragmentaires d'un Nijinski photographique. Le Faune est tordu, gauchi par la dissymétrie qui fait de lui le composé de deux corps étrangers l'un à l'autre: Nijinski et Chouinard (et aussi Chouinard et Prieur...). Ces surimpressions de contours hétérogènes semblent aussi dessiner une partition entre vrai-corps (corps naturel) et faux-corps (prothèse), et la grossièreté de l'artifice semble assurer 
d'emblée une certitude: la danseuse-Prieur danse avec son vrai-corps, même s'il est par endroits recouvert de prothèses. Son entrée, au début, laisse apercevoir cette jambe manifestement étrangère ou rapportée, qui s'ajuste mal aux contours réguliers et à nouveau symétriques du buste. Pourtant, le travail du geste vient troubler et contredire cette certitude première. Selon les appuis, les contrappostos instables, les tentatives toujours mises en échec du bassin pour se verticaliser au-dessus des jambes, les rapports d'appartenance ou de continuité entre le buste et «la vraie jambe» tendent à s'inverser. La fausse jambe (la jambe faussée dans ses contours) pourrait bien être la véritable jambe du corps-Faune. La vraie jambe pourrait bien en être un appendice rapporté. Ce n'est pas la vérité du contour du corps qui fait la justesse du mouvement; c'est la justesse du mouvement qui fait la vérité du corps. Le corps, y compris ses appendices démontables.

Les photos du baron de Meyer, posées et prises en studio, font partie de l'héritage mythique d'un Nijinski dont aucune trace mouvante (filmée, par exemple) ne subsiste. Nous savons qu'elles sont, dans leurs plus fins détails, des images «telles que les a voulues» Nijinski. La technique photographique travaille la matière et la texture de l'image, substituant peut-être le grain de l'image au grain du geste de Nijinski, ici arrêté pour les besoins de la prise de $v{ }^{16}$. La composition des images prolonge les lignes de force qui composent et recomposent le corps du danseur. Le Faune de Chouinard entre dans les contours de ces photos, «il montre les différentes façons d'entrer dans la posture» ${ }^{17}$, s'y ajuste, en sort, y retourne; il réinvente l'histoire de chaque posture, il agite de son propre mouvement les images arrêtées de Nijinski. Il dédouble sa propre identité: la mimétique des contours morphologiques et posturaux dessine le fantôme d'un Nijinski d'affiche, figure tutélaire de toute la danse moderne; l'impossibilité de tenir les figures dont le Faune-Chouinard ne cesse de se retirer surimpose un autre corps, remuant, instable, agacé par les tensions égales d'un désir ou d'une souffrance qui ne se décident jamais en faveur d'une orientation.
Dans le ventre, la gorge et la bouche, se rencontrent des forces contradictoires dont aucune ne prend le dessus sur l'autre, laissant le corps dans son trop-plein de tensions qu'aucune fonctionnalité ne vient délier. Cette tension improductive n'est pas là seulement pour démonter les fondations du corps-organique, créant une gêne que chaque spectateur semble interpréter différemment (du sentiment d'impuissance à celui de la surpuissance, de la résolution orgasmique à l'impossibilité de la décharge, de l'ironie à la trahison de l'œuvre d'origine) en fonction sans doute de ses propres repères en matière d'orga(ni)sme. Elle met aussi en tension une vieille histoire de danse: le rapport à la forme et au signe à produire.

J'avais abandonné les cours de classique et je continuais à m'entraîner quotidiennement à la barre. Et c'est en faisant les exercices les plus académiques que tout à coup, des gestes totalement incongrus ont commencé à m'échapper. J'ai bien tenté d'abord de revenir à la normale, mais ça a été plus fort que moi. ${ }^{18}$

La figure imposée, prothèse du corps dansant: voilà peut-être la véritable-origine-de-la-danse. La figure à remplir sans laquelle la métamorphose du corps en vrai-danseur ${ }^{19}$ ne saurait advenir. L'objet-corps dont il faut «évacuer les excroissances, les bourgeonnements, les poussées, les courbures, les irrégularités aléatoires et fluctuantes» afin de l'insinuer dans la-figure-de-danse. Et l'objet-corps qui indéfiniment fait le danseur par débordements, excroissances, gauchissements. Il n'y aurait pas de Faune-Chouinard sans les contours du monument imaginaire Nijinski. Il n'y aurait pas non plus de Faune-Chouinard sans ces excroissances, ces résistances, ces déviances qui lui rendent la figure de Nijinski intenable.

Pourtant le discours de Chouinard semble décrire un cheminement du geste quelque peu différent de ce que je crois voir dans son Faune. "C'est pour ça qu'on danse: pour l'occasion de cette intensité... C'est comme un autre corps qui croît depuis l'intérieur du vôtre", dit-elle encore. Le paradoxe du mouvement que décrit Chouinard, cette poussée de l'intérieur qui métamorphose le corps en une autre vérité, fait écho à de nombreuses idéologies du corps dansant 
(notamment moderne) que j'appellerai corps-desprofondeurs. Révélation d'une intériorité, ou encore épluchage, élagage de couches superficielles ${ }^{20}$ qui viendraient masquer ou empêcher le geste profond, ces idéologies construisent un corps où l'extériorité se doit d'être l'exact reflet des intérieurs, sous peine de passer pour un mensonge. Mais, contrairement à ce que Chouinard semble revendiquer, sa danse ne révèle rien d'intérieur qui viendrait recouvrir ou dissoudre les effets de surface; son Faune appartient à l'ordre du monstre - corps composé d'éléments d'ordres hétérogènes, voire contradictoires ${ }^{21}$, ou encore à l'ordre de la sorcière, dont les humeurs inquiétantes ont souvent servi de motif à la danse moderne réinventant des corps «autres» 22 .

Je porte un costume et non un vêtement. C'est pour rendre accessible aux spectateurs les transformations qui se sont opérées à l'intérieur de mon corps au cours de la création de la pièce. Le costume est une sorte d'amplificateur qui sert à projeter cette transformation. 23

La prothèse porte des enjeux de transformations; pourtant, la voir comme une émanation corporelle, c'est réduire ces enjeux à une fonctionnalité naturaliste. Chouinard met en vibration bien autre chose: toute la gamme, d'abord, qui va de l'organe à l'inerte et à l'artificiel, et surtout ce qui est "entre" l'organe et l'artifice; toutes les modulations de ce qui est à la fois l'un et l'autre: décoration, appendice, greffe, mutations, transgenres. Elle met en mouvement ces objets dont Preciado a si efficacement décrit le rôle: torpiller la catégorie du corps, et délier organes, sexes et modes de jouissance des normes de l'anatomie fantasmatiquement naturelle. Mais tandis que Preciado use de l'objet pour faire exploser le corps compris comme contours, sexuation et fonctionnalité, Chouinard fait exploser la prothèse comprise comme objet. En recomposant son corps en prothèses multiples - les-objets, la-figure-de-danse, le-faune-de-Nijinski, le-corps-de-Chouinard, le-corps-de-l'interprète... -, elle délie la prothèse de son statut d'objet et lui ouvre le champ du sensible: elle module les états de chair de l'artifice. La prothèse n'a pas d'existence en soi, mais seulement dans son rapport à un geste. Comme le corps dansant est construit et réinventé par le geste, la prothèse est construite, réinventée et défaite par le geste dansé. Il n'y a pas de corps sans prothèse. Il n'y a pas de prothèse sans geste.

\section{FLOTTEMENTS DE FAUNES}

Novembre 99: longue litanie des solos de Chouinard et leur avalanche d'images, de son, d'objets, de corps. L'ensemble retient mon attention parce que je ne sais pas quoi en penser. Quelques jours (ou semaines?) plus tard, au cours d'une discussion avec des étudiants, je leur parle de la bande-son du Faune, pierre de touche de ma «lecture" (ou non-lecture) de la pièce. Personne n'en a gardé un souvenir marquant. Aije inventé ce son imaginaire? Je ne connais rien de Chouinard, si ce n'est quelques textes épars, glanés depuis ce premier contact avec son travail. Mes tentatives pour prendre contact avec la compagnie échouent. Est-il possible de penser/écrire sur le travail d'une artiste contemporaine sans avoir eu de contact avec elle? Je mesure à quel point ma pensée sur les œuvres est assujettie à un lien (toujours fantasmatique) avec le corps du chorégraphe. Finalement, j'obtiens une vidéo du Faune. Surprise: je reconnais les formes, mais je ne reconnais pas la pièce. Quel travail aura-t-elle fait dans ma mémoire depuis cette première rencontre fugitive? Surprise double: la bande-son de mon souvenir - souffles, mugissements, bruits mécaniques ironiques - est en fait la classique musique de Debussy. J'avais peut-être vraiment rêvé ce son démoniaque? Nouvelles excavations documentaires. Depuis le début, j'appelle tout cela «le Faune»; mais deux titres semblent diffusés dans les documents de la compagnie: L'Après-midi d'un Faune, et Prélude à L'Après-midi d'un Faune. Explication: la première version, en 1987, dansée par Marie Chouinard, était accompagnée par la fameuse bande-son que j'ai retenue.

En 1994, Marie Chouinard «transmet» pour la première fois un de ses solos en reprenant, pour les danseuses de sa compagnie, sa chorégraphie L'Après-midi d'un Faune [...] Ce faisant, Marie Chouinard crée Prélude à L'Après-midi d'un Faune, une œuvre chorégraphiée cette fois à partir de l'ouvre de Claude Debussy. 24 
La version que j'ai vue était pourtant dansée par l'une «des danseuses de la compagnie», accompagnée de la bande-son et de son titre dépourvu de prélude. Explication (bis): pour le programme de tous ses solos, Chouinard aurait choisi de reprendre les versions "originales». Enfin, je croise les textes d'Ann Cooper Albright où la pièce apparaît sous le titre La Faune, dont je ne trouve aucune trace dans les documents de la compagnie que j'ai pu collecter. Albright a vu le solo au moment de sa création, en 1987. Je soupçonne que ce premier titre aura été remplacé peu après la première.

Je n'ai jamais revu la pièce sur scène. Le travail sur la version vidéo qui m'est parvenue ne parvient pas à effacer l'impression produite par la première bandeson. Je ne saurai jamais si la chorégraphie a été modifiée ou pas lors du changement de musique. Carole Prieur, interprète du Faune de la vidéo, demeure mon interprète du Faune (je ne sais pas laquelle, de Prieur ou Marie-Josée Paradis, dansait la représentation que j'ai vue). Je travaille à partir d'un fantôme recomposé d'une pièce qui, manifestement, ne se reconnaît que des duplicatas, tous semblables, et tous dissemblables. Et comme le corps de ce Faune qui n'a cessé d'inquiéter ma pensée, le corps de mon fantôme chorégraphique résiste à toute saisie. Tandis que les «fondements» de mon analyse s'évanouissent, vacillent ou se confondent, ma lecture se disperse; les images superposées diffractent à ses prémisses chaque système de lecture. Comme le corps du FauneChouinard, habité par nombre de corps-poncifs hétérogènes, ce texte flotte, entre le discours-de-moi et divers discours-des-autres contradictoires. Comme le Faune-Chouinard, j'adhère à certains de ces discours, à d'autres pas, sans pour autant pouvoir (vouloir?) les faire taire. Je laisse leurs flux sans origine faire muter le cours du texte et le corps des lettres.

À partir de quel degré de «réalité» ou de certitude quant à l'œuvre, un projet critique peut-il s'estimer légitime? Ou encore: quelle est la nature d'un projet critique qui ne s'efforce pas de recomposer une certitude de l'œuvre? L'Après-Midi de la Faune: une prothèse qui fait exploser la machine critique.

\section{N O TES}

1. L'ensemble de ces réflexions doit beaucoup à nombre de discussions avec l'équipe du département Danse de Paris VIII : Hubert Godard et Isabelle Launay, ainsi qu'aux étudiants de maitrise et DEA de ce même département, participants de «l'atelier critique ", dont les réflexions et études sur ce solo ont largement nourri les miennes. Et un clin d'œil amical aux travaux de Beatriz Preciado, qui sont venus à point nommé aider à articuler quelque chose du rapport à l'objet qui me semble au cœur du Faune de Chouinard.

2. B. Preciado, Manifeste contra-sexuel, Paris, Balland, 2000, p. 71.

3. A. Suquet, L'Émoi et la métamorphose : le corps selon Marie Chouinard, texte inédit, New York, 1998.

4. M. Chouinard, citée par A. Suquet, ibid.

5. Parmi les mouvements de réhabilitation du statut de l'interprète, on pense notamment, en France, aux travaux du Quatuor Knust, précisément autour du Faune de Nijinski, qui mettent en évidence l'illusion d'une quelconque "vérité de l'œuvre " par la mise en regard de multiples «incorporations » de la partition chorégraphique par des interprètes tous plus différents les uns que les autres, et rendent visible l'historicité singulière de chaque construction interprétative.

6. Pour une réflexion approfondie sur la question de la formation du danseur et les "disciplines du corps " dans la danse, voir dans ce même numéro de Protée l'article d'Isabelle Launay, «Le don du Geste».

7. Voir à ce sujet les reproches d'H. Gauville dans « Du Chouinard Canada dry ", Libération, vendredi 5 novembre 1999.

8. B. Preciado, op. cit., p. 119.

9. A. Lepecki, "Undoing the fantasy of the dancing subject: "still acts", dans Jérôme Bel's "The Last Performance" ", travail pour le séminaire "Choreographing History ", Lyon, septembre 2000.

10. La critique politique des rapports chorégraphe-interprète comme rapports de pouvoir est en bien des points comparable à la longue série des critiques bipolaires féministes, de genre, culturelles, etc. Certains textes récents mettent à mal l'existence des catégories homme/femme, gay/hétéro, etc., comme entretenant cela même qu'elles prétendent dénoncer (voir notamment B. Preciado, op. cit.). Les discours sur «le statut de l'interprète", en consolidant la catégorie, n'en consolident-ils pas du même coup les emprises?

11. A. Suquet, op. cit.

12. Selon l'expression de S. Prokhoris, Le Sexe prescrit, Paris, Aubier, 2000.

13. Pour une analyse détaillée des rapports de genre et des jeux du désir masculinféminin dans le solo, voir A. Cooper Albright, Choreographing Difference, Hanover, Wesleyan University Press, 1997.

14. C'est d'ailleurs ce que propose H. Gauville: «[...] les éléments ajoutés (décors, costumes, musiques, paroles....) sont gênants. Trop souvent d'un mauvais goût que rien ne justifie, ils finissent par brouiller la lisibilité de la danse " (op. cit.).

15. B. Preciado, op. cit., p. 121

16. Voir N. Yokel, Un Regard sur le Faune : l'analyse de "L'Après-Midi d'un Faune", d'après les photographies d'Adolph de Meyer, mémoire de maîtrise, département Danse de Paris VIII, septembre 1997.

17. H. Godard, conversation autour de la vidéo de Chouinard.

18. R. Boisseau, "Marie Chouinard, chamane moderne de la danse québécoise», Le Monde, 5 novembre 1999.

19. I. Launay, op. cit.

20. D. Bagouet notamment avait recours à cette métaphore pour parler de sa formation de danseur, du passage vers la danse contemporaine, ainsi que pour certains moments-pivots dans son œuvre chorégraphique.

21. Dans une réflexion sur les différentes catégories de monstres de notre culture,

I. Autran mentionne les malformations physiques présentées comme «infrahumaines " : hommes-lions, hommes-homards, etc., qui suscitent, dit-elle, une « incertitude intellectuelle». Une autre forme de monstruosité serait celle produite par l'imaginaire mythologique, qui fait des monstres (Titans, Titanides, Cyclopes, etc.) les figures de "la période pré-humaine où régnait le chaos». (I. Autran, «Monstruosités du corps", dans "Corps ", Prétentaine, n $12-13$, mars 2000).

22. Voir à ce sujet I. Launay, «Portrait d'une danseuse en sorcière: Hexentanz de Mary Wigman ", Théâtre/Public, n 154-155, 2000. La figure de la sorcière est souvent (positivement ou négativement) évoquée à propos de danseuses-chorégraphes modernes - notamment Chouinard - dont les corps étranges (ou la puissance?) inquiètent la critique.

23. M. Chouinard, «Entretien ", Les Vendredis du Corps, Cahiers de Théâtre Jeu/

Festival international de Nouvelle Danse, Montréal, 1993.

24. Texte de communication de la compagnie. 